\title{
Positron Annihilation in Metals Defected by Action of the Tensile Force
}

\author{
W. Rudzińska*, J. Pająk, M. Szuszkiewicz, G. Bujnarowski \\ AND A.A. KLUZA
}

Institute of Physics, Opole University, Oleska 48, 45-052 Opole, Poland

Results of experimental investigations of uniaxially elongated mono- and polycrystalline samples of several metals (Fe, $\mathrm{Ta}, \mathrm{Pd}, \mathrm{Ag}$, and $\mathrm{Au}$ ), performed using the positron annihilation methods, are reviewed. The dependences of the $S$-parameters and positron lifetimes on the relative elongation of the samples were presented. The data obtained for polycrystalline samples indicate that in the proportionality and limited proportionality regions the changes in the physical properties are governed mainly by generation of vacancies and by kinetics of formation and transformations of vacancy clusters occurring first of all on the grain boundaries of monocrystallites. In the region of plastic deformations the dominant defects are dislocations and vacancies and their aggregates generated due to the formation and movement of the dislocations of the primary and secondary slip. In the case of monocrystalline samples it was found that the dynamics of the dislocations and vacancy generation during the sliding of some crystallographic planes depends on the crystallographic direction.

PACS numbers: $78.70 . \mathrm{Bj}$

\section{Introduction}

It is well known that the ability of metals to be deformed under action of external forces results from the generation of crystal lattice defects, mainly dislocations. Analytical description of the process of deformation of uniaxially extended A1, A2, and A3 type metals is given, among others, in papers [1-4].

The behaviour of polycrystalline samples in the process of deformation by uniaxial tension differs from that of monocrystalline samples. It is generally known that poly- and monocrystalline metal samples are deformed under the influence of increasing external forces, first elastically and after exceeding an elasticity limit, the deformation starts to be plastic. The polycrystalline metal sample is an ensemble of microcrystallites with randomly oriented crystal axes. Even in the equilibrium state, the grain boundary regions are strongly defected. After exceeding

*corresponding author; e-mail: wiesia@uni.opole.pl 
some crystal strain the defects accumulated at the grain boundaries penetrate through the grain boundaries. In the macroscopic scale this results in appearance of the lower and upper limits of elasticity. In this interval the strain oscillates around some value proportional to the energy required for penetration of the grain boundary by the defect. In this interval some ordering of the intergrain regions is observed.

After equalization of the concentration of dislocations in all the grains, their penetration through the grain boundaries becomes unimportant. At this moment the plasticity arrest ends and the plastic range starts. The dislocations (especially the edge dislocations) strongly interact among themselves and the hardening of the sample starts. Although the cross section area of the sample decreases uniformly along the sample length, the strain increases rapidly because of the hardening of the sample. In this range the mutual displacements of the grains, their coalescence because of joining small grains, and decomposition of big grains into small pieces is observed.

The dislocation movement in polycrystalline materials is blocked by grain boundaries and therefore the easy slip stage, observed in monocrystals, does not occur in the case of polycrystals. This blocking effect of grain boundaries is the reason for simultaneous initiation of plastic deformation in various slip planes. Therefore, the elasticity limit for polycrystalline materials is much higher than that for monocrystals, the increase in the value of elasticity limit being the larger the smaller are the grain dimensions. The obstacles for the dislocation movement are mainly the broad-angle grain boundaries, whereas the low-angle grain boundaries are permeable for the mobile vacancies.

During the elastic deformation of the monocrystalline metal samples the ordering of the crystal lattice remains unchanged, only the interatomic distances are changing. This leads to a change in the volume of elementary lattice cell. If the value of external forces exceeds the extremum of interatomic forces, metals start to deform plastically. Plastic deformation requires the dislocation movement by gliding in the slip plane or by climbing [5]. During the climbing process the edge (or mixed) dislocations are moving by adjoining or releasing vacancies to or from the extra half plane. This way the edge and mixed dislocations are the sinks (or sources) for vacancies. The slip starts when the tangential stress in the easy slip plane and easy slip direction (close packed planes and directions), caused by external forces, reaches some threshold value - the so-called critical tangential stress $\left(\tau_{\text {cr }}\right)$. The dependence of the tangential stress $\tau$ in the slip direction on the elongation force $F$ is described by the relation $[2,3,5]$ :

$$
\tau=\frac{F}{S_{0}} \cos \lambda \cos \phi,
$$

where $F$ is the elongation force, $S_{0}$ - the cross-sectional area of the elongated sample, $\lambda$ - the angle between slip direction and force $F, \phi-$ the angle between $S_{0}$ plane and slip plane. 
The value of $\tau$ depends on the orientation of monocrystal with respect to the force $F$. The $\tau$ attains maximum when the angle $\phi$ between the sample axis and the slip plane and slip direction is $45^{\circ}$. For $\phi=90^{\circ}$ or $0^{\circ} \tau$ is 0 . In the case of gold the easy slip planes are $\{111\}$ planes and the easy slip directions are (110) and (100) directions. The processes occurring during the plastic deformation are studied mainly by microscopic techniques. It is well known that during the dislocation movement the vacancies are created due to the sliding of some crystallographic planes. The possibilities to observe the behaviour of these vacancies by the microscopic techniques are rather limited. This aspect of mechanical deformation can be, however, very effectively studied by the positron annihilation methods.

\section{Results and discussion}

The present paper is aimed to summarize the results of hitherto performed positron annihilation studies of structural changes occurring in mono- and polycrystalline samples of different metals during the static tensile tests [5-11]. In paper [5] angular distributions of the positron annihilation quanta were determined for pure and hydrogenated palladium samples. The data are presented as the relative difference curves, with respect to the undeformed sample, determined for pure and hydrogenated palladium samples extended up to six different elongation degrees $(0.39 \%, 0.77 \%, 1.5 \%, 1.9 \%, 2.7 \%$, and $3.5 \%)$. It was found that for relative elongations ranging from 1.9 to $3.5 \%$, in both the pure and hydrogenated palladium, the positrons are trapped in defects larger than vacancy clusters. The values of the $R$-parameter for pure palladium samples elongated up to $0.77 \%$ and $1.5 \%$ indicate a dominant role of smaller defects, like the vacancy clusters.

The measurements of angular distribution of positron annihilation quanta, positron lifetime spectra and resistivity for iron samples were presented in [6]. It has been found that the course of the dependence of the $S$-parameter on the relative elongation $\varepsilon$ is nonmonotonous. The correlation between the $S$ - and $W$-parameter values suggests that positrons are trapped in three different types of defects. It is obvious that elongation of the sample generates dislocations, grain boundaries, as well as point defects - vacancies and interstitials. It is difficult to judge which of them dominate in trapping the positrons in an investigated sample. The results presented in [6] indicate that the changes in the positrons lifetime parameters correspond well to the changes in the $S$-parameter value as well as with the changes in the internal strain and in the electrical resistivity in the investigated range of elongations $0<\varepsilon<0.6 \%$.

In order to bring into evidence the common features of the processes occurring during the static tensile test, the dependences of the annihilation parameter $S$ on the relative elongation $\varepsilon$ all the investigated metals ( $\mathrm{Ta}, \mathrm{Fe}, \mathrm{Ag}$ ) [7] are shown together in Fig. 1.

The analyse of the differences in the $S$-parameter value is performed with respect to that for unloaded sample. It is reasonable to assume that the initial 


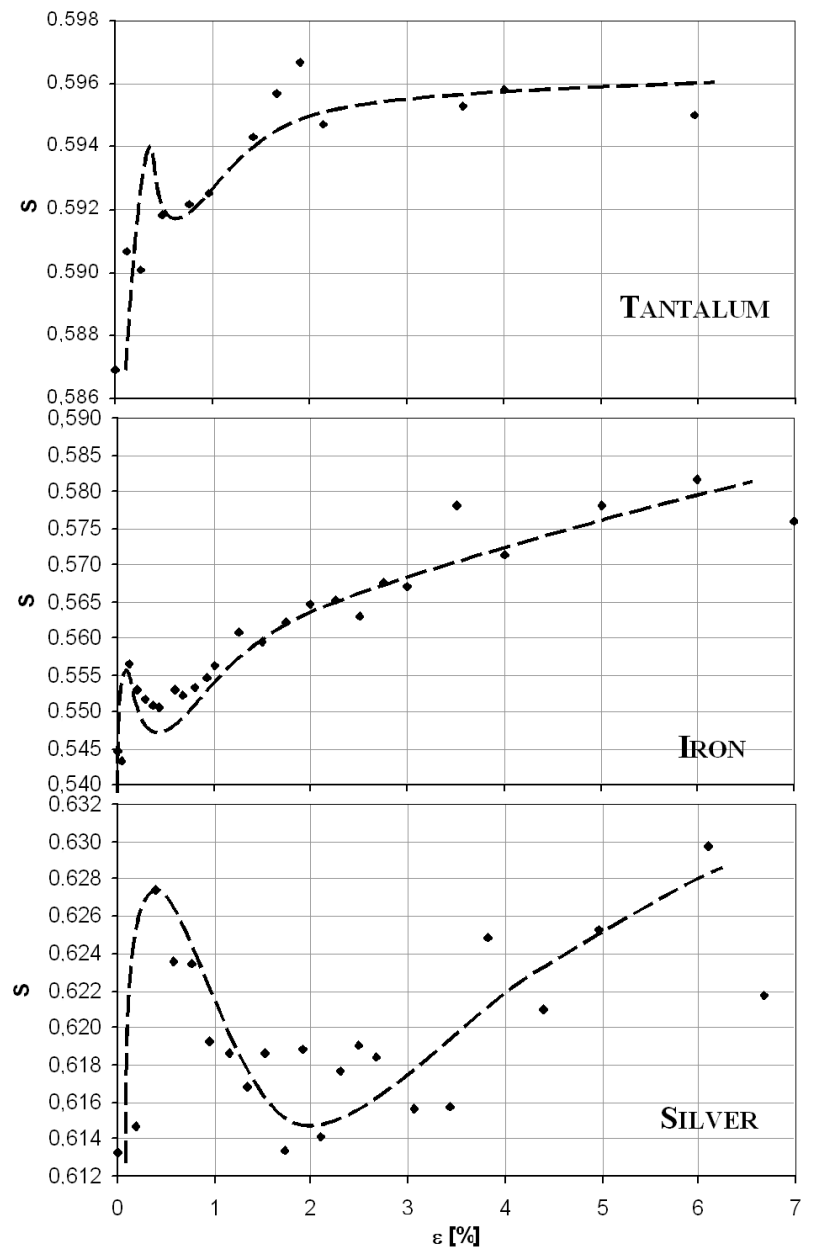

Fig. 1. Dependence of the $S$-parameter on the relative elongation $\varepsilon$ for tantalum, iron, and silver samples (from [7]).

increase in the $S$-parameter value is caused by appearance of new positron trapping defects like vacancies and their agglomerations. With a further loading of the samples the concentration of nonequilibrium vacancies decreases because of the activation of dislocations and movement of crystalline planes in microcrystallites along the easy slip planes. Therefore, the $S$-parameter decreases up to some minimum value. The further loading of the sample increasing the concentration of dislocations does not compensate the effect of decreasing vacancy concentration.

Location of the minimum value of the $S$-parameter can be ascribed to the region of the maximum mobility of dislocations - the elasto-plastic range in the strain-stress curve. A further increase of the sample load causes a further increase in the concentration of dislocations, and their blocking both in the volume of 
grains and at their boundaries. Therefore, the mobility of dislocations decreases and simultaneously the internal stress increases. This in turn causes an increase in the vacancy concentration resulting in an observed increase in the $S$-parameter value up to some saturation level.

Figure 2 represents the correlation between the $S$ - and $W$-parameter [7] values determined for each of the relative elongation values. Two straight lines are used to fit the experimental points for each of the investigated materials. One of these straight lines fits the points corresponding to the elongation interval $0<\varepsilon<\varepsilon_{\min }$. The remaining points lie in the close vicinity of the second straight line. Two straight differently sloped lines mean that positrons are trapped in two different types of defects. As it follows from Fig. 2, in different ranges of uniaxial tension different types of defects play a dominant role in positron trapping in investigated materials.
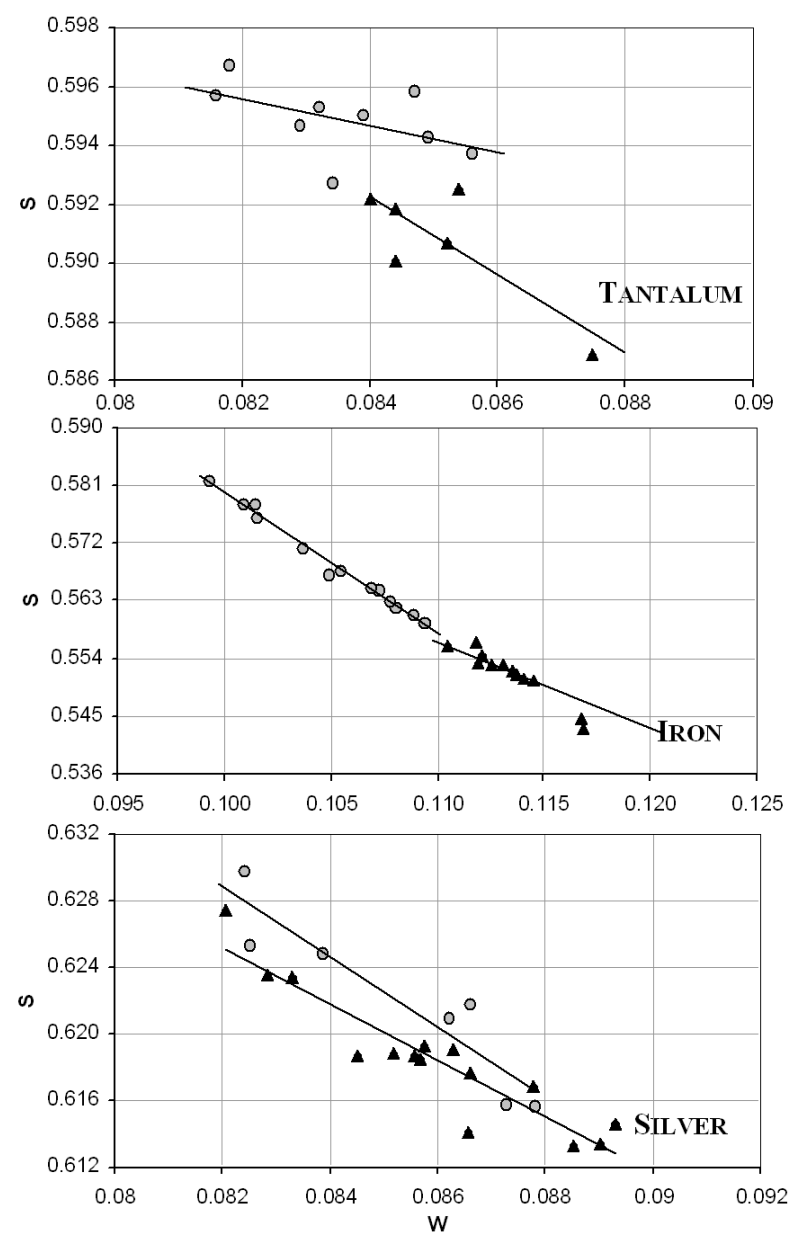

Fig. 2. Correlation between the $S$-parameter values for three investigated metals (Ta, $\mathrm{Fe}, \mathrm{Ag}$ ) (based on [7]). 
In the range of elasto-plastic deformation the vacancies and their aggregates generated mainly in the grain boundary regions play the dominant role. In the remaining ranges of the strain-stress curve the dominant role belongs to the vacancy type defects formed due to the generation and movement of dislocations in the primary and secondary slip.

The detailed investigations of the angular distributions of the positron annihilation quanta, and positron lifetime were performed for poly- and monorystalline silver and gold samples defected by uniaxial tension [8-11]. The dependences of the $S$-parameter on the relative elongation and on the $W$-parameter for monocrystalline gold samples oriented in the (110) and (111) directions are presented in Fig. 3 [8].

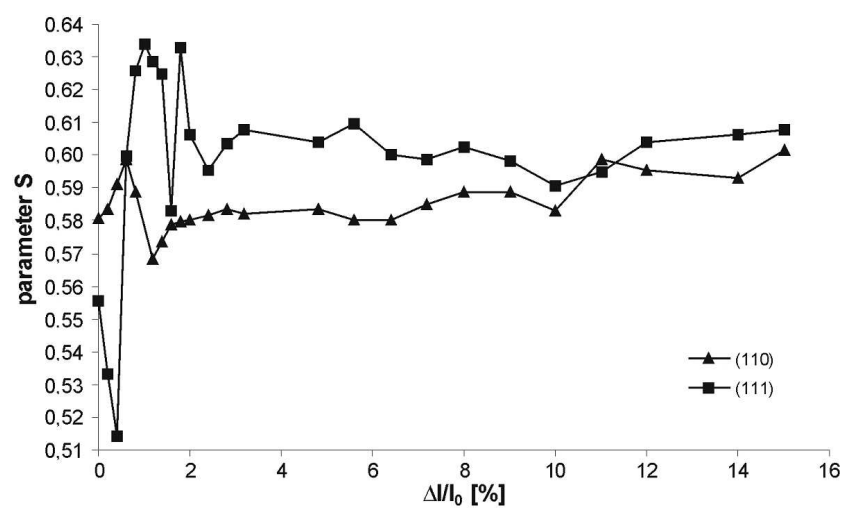

Fig. 3. The dependence of the $S$-parameter on the relative elongation of the gold samples oriented in (110) and (111) directions (based on [8]). $\Delta l / l_{0}=\varepsilon-$ relative elongation of the sample.

The orientation of the sample in relation to the tensile force means that the tensile force is parallel to the plane perpendicular to a given direction. In the case of our experiment they are $\{110\}$ and $\{111\}$ planes. For the sample oriented in (110) direction, the angle between easy slip plane that is the $\{111\}$ plane and the tensile force is $45^{\circ}$. In agreement with formula (1), in order to reach the critical value of the tensile force, the internal stress in the sample increases before every next slip. As a result of increase of the internal stress, dislocations and vacancy-type defects are formed. After reaching the critical value of the tangential stress, the slip is activated in $\{111\}$ planes. After finishing the slip, the sample is easing of the tension partly, and densities of dislocations and vacancies decrease. The jump like character of the process of set going of slip on easy slip plane is visualised by characteristic increase and decrease in the course of $S$-parameter for monocrystalline samples oriented in (110) direction. In the case of the sample oriented in (111) direction, the tensile force is parallel to the $\{111\}$ plane. In agreement with formula (1), the tangential stress in the easy slip plane $(\{111\}$ 
plane) equals 0 . The slip can occur in the other planes, less favourably oriented in relation to the tensile force that needs a larger value of the critical tangential stress to activate the slip. Because of the above-mentioned reason, the changes in the course of $S$-parameter for sample oriented in (111) direction are much larger than those for sample (110) and the peaks with the largest value of the $S$-parameter are shifted to the higher value of the relative elongation.

For both directions the dependence of the $S$-parameter on the $W$-parameter can be fitted by one straight line that means that there are the traps that cause the same influence on the annihilation parameters.

In paper [8] angular correlation curves of the positron annihilation quanta have been measured for monocrystalline gold samples, oriented in (110) and (111) directions, submitted to increasing relative static uniaxial elongation. The dependences of the $S$-parameter on the relative elongation of the gold samples and $S$-parameter on the $W$-parameter correspond well with those observed for monocrystalline silver samples [9]. Similarly as for silver, in the case of gold the positron traps are, most probably, dislocations and vacancies generated during the plastic deformation by elongation.

The measurements of the positron lifetime for uniaxially elongated polycrystalline silver sample were performed, too [10]. The main results of these investigations are shown in Figs. 4, 5. Up to 0.5\% elongation, a decrease in mean value of the positron lifetime is observed. Similarly as in the case of $S$-parameter, this is probably connected with changes in the volumes of unit cells of the crystalline lattice due to the deformation as well as with changes in the grain boundaries under the action of external tensile force. With a further elongation the plastic deformation of the sample begins due to the dislocation movement. The grain boundaries are very effective obstacles for dislocations movement on which the accumulation and association of dislocations takes place. This leads to an increase in the local stress, which in turn may act as dislocation source in adjacent grains. All of these processes cause changes in the defect structures of investigated samples, including the concentration of crystal lattice defects (dislocations vacancies and their agglomerations). This finds confirmation in the increase in the mean value of the positron lifetime with increasing elongation of the sample. Figure 4 represents the dependence of the positron lifetime $\tau_{2}$ of the long-living component of the elongation of the sample. Figure 5 represents the dependence of the intensity of the long-living component of the positron lifetime spectra on the relative elongation of the sample. In the positron lifetime spectra for well annealed sample the long-living $\left(\tau_{2}=275 \mathrm{ps}\right)$ component has a relative intensity of $\sim 14 \%$. After elongation of the sample by $0.5 \%$ the value of $\tau_{2}$ decreases to 245 ps. During the elongation of the sample the vacancies are generated and agglomerate due to migration processes. The $\tau_{2}$ value remains almost constant up to elongations of about $2.5 \%$, but its intensity rapidly increases to $32 \%$. A further elongation of the sample is accompanied by an increase in the $\tau_{2}$ value up to about 260 ps at $9 \%$ 


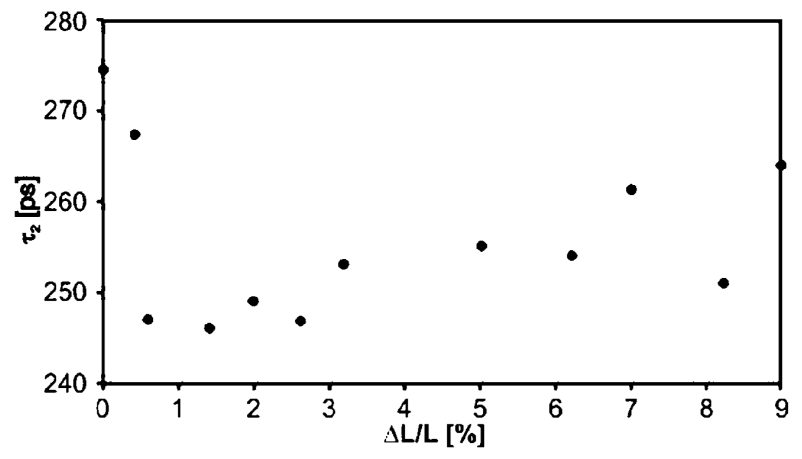

Fig. 4. The dependence of the lifetime of the long-lived component $\tau_{2}$ of the positron lifetime spectra on the relative elongation of the sample (based on [11]). $\Delta L / L=\varepsilon$ relative elongation of the sample.

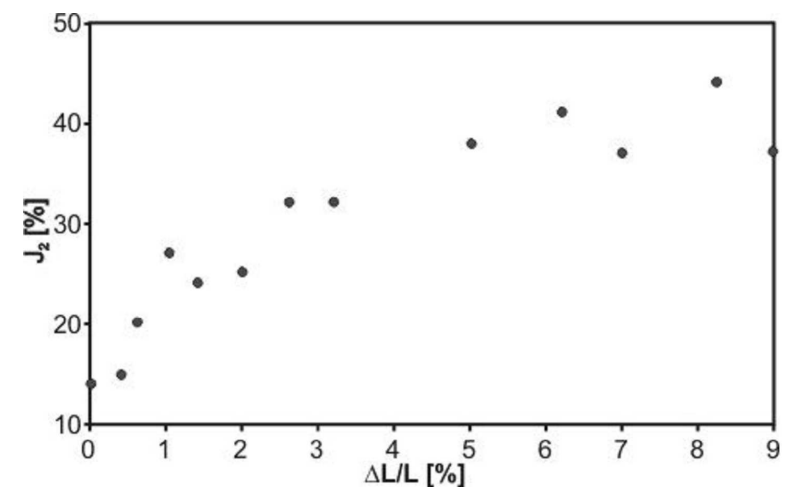

Fig. 5. The dependence of the intensity of the long-lived component $J_{2}$ on the relative elongation of the sample (based on [11]). $\Delta L / L=\varepsilon$ - relative elongation of the sample.

elongation. The intensity of this component increases up to $40 \%$ at $6 \%$ elongation and does not change with a further elongation of the sample. The last effect can be ascribed to the saturation of positron annihilation in vacancy clusters.

\section{Conclusions}

From the investigations performed in the frames of the present study for several methods, one can conclude that the positron annihilation methods are very useful as methods supporting the static tensile tests. The changes in the positrons annihilation parameters caused by uniaxial static elongation permit to reveal and to describe the microscopic changes in the structure of the investigated materials, and enable us to follow the kinetics of generation of defects in conjecture to the particular ranges of the strain. 


\section{References}

[1] R.W.K. Honeycomb, The Plastic Deformation of Metals, 2nd ed., E. Arnold Ltd., London 1984.

[2] W. Gambin, Engng. Trans. 39, 303 (1991).

[3] K. Przybyłowicz, Structural Aspects of Metal Deforming, Warsaw University of Technology, Warsaw 2002 (in Polish).

[4] W. Gambin, K. Kowalczyk, Plasticity of Metals, Warsaw University of Technology, Warsaw 2003 (in Polish).

[5] W. Rudzińska, J. Pająk, Cz. Szymański, R. Szatanik, Acta Phys. Pol. A 99, 479 (2001)

[6] W. Rudzińska, J. Pająk, M. Szuszkiewicz, G. Bujnarowski, A.A. Kluza, Phys. Scr. 71, 111 (2005).

[7] W. Rudzińska, J. Pająk, M. Szuszkiewicz, G. Bujnarowski, A.A. Kluza, Acta Phys. Pol. A 107, 857 (2005).

[8] J. Pająk, W. Rudzińska, M. Szuszkiewicz, M. Garłowska, Acta Phys. Pol. 107, 848 (2005).

[9] J. Pająk, W. Rudzińska, Cz. Szymański, S. Gąsior, K. Malisz, in: Proc. 34th Polish Seminar on Positron Annihilation, Ed. K. Jerie, University of Opole, University of Wrocław, Opole 2002, p. 55.

[10] W. Rudzińska, M. Szuszkiwicz, A.A. Kluza, G. Bujnarowski, J. Pajạk, Chemia i Iżnynieria Środowiska 10, 157 (2005).

[11] J. Pająk, W. Rudzińska, R. Pietrzak, Cz. Szymański, W. Smiatek, Phys. Scr. 67, 186 (2003). 